\title{
The enigma of lithium in roAp stars
}

\author{
N. Polosukhina ${ }^{1}$, A. Shavrina ${ }^{2}$, N.A. Drake ${ }^{3,4}$, V. Tsymbal ${ }^{5}$, \\ M. Hack ${ }^{6}$, P. North $^{7}$, V. Khalack ${ }^{2}$, J. Zverko $^{8}$, J. Žižňovský ${ }^{8}$ \\ and Ya. Pavlenko ${ }^{2}$ \\ ${ }^{1}$ Crimean Astrophysical Observatory, Ukraine \\ email: polo@crao.crimea.ua \\ ${ }^{2}$ Main Astronomical Observatory, National Academy of Science, Ukraine \\ email: shavrina@mao.kiev.ua \\ ${ }^{3}$ Observatório Nacional/MCT, Rio de Janeiro, Brazil \\ email: drake@on.br \\ ${ }^{4}$ Sobolev Astronomical Institute, St. Petersburg State University, Russia \\ ${ }^{5}$ Simferopol State University, Ukraine \\ email: vad@ccssu.crimea.ua \\ ${ }^{6}$ Department of Astronomy, Trieste University, Italy \\ email: hack@ats.stro.it \\ ${ }^{7}$ Institut d'Astronomy, Université de Lausanne, Switzerland \\ email: pierre.north@obs.unige.ch \\ ${ }^{8}$ Astronomical Institute, Slovak Academy of Science, Slovakia \\ email: zve@ta3.sk
}

\begin{abstract}
An internacional project "Lithium in magnetic Ap stars" has been run since 1996 with the purpose of creating an observational database allowing systematic studies of the abnormal occurence of lithium on the surface of cool Ap stars. The $2.6 \mathrm{~m}$ telescope at the Crimean Astrophysical Observatory, the ESO CAT, ESO 1.52 m telescope with FEROS, the Nordic Optical Telescope, and the 74" telescope at Mount Stromlo were employed to collect observations at the regions of the lithium resonance lines $\lambda 6103$ and $\lambda 6708 \AA$.

Observations of the roAp-stars HD 83368, HD 60435 and HD 3980 revealed considerable periodical Doppler shifts of the line of lithium $\lambda 6708 \AA$ which can be explained by the presence of lithium spots on their surfaces. Conjunction with structures of the magnetic field is apparent.

A detailed study of the blend at $\lambda 6708 \AA$ in HD 101065 confirmed the anomalous overabundance of lithium amounting to $3.1 \mathrm{dex}$, as well as unusual isotopic ratio ${ }^{6} \mathrm{Li} /{ }^{7} \mathrm{Li} \sim 0.3$.
\end{abstract}

Keywords. Stars: chemically peculiar, stars: Li abundance

\section{Introduction}

The lithium problem in CP stars has been, for a long time, a controversial subject. Even the existence of the $\operatorname{Li}$ I $6708 \AA$ line in the spectra of roAp stars was in doubt. The behaviour of the Li I $6708 \AA$ line in CP stars is very puzzling. In some stars this line is a strong and variable feature, in others, with the same physical parameters, it is not detectable at all. It is quite probable that both atomic properties of lithium, its low ionization potential and its low mass, are important ingredients that make Li ions to be subject of strong hydrogen ambipolar diffusion (Babel \& Michaud 1991; Babel 1993) what can significantly enhance the surface $\mathrm{Li}$ abundance and also the ${ }^{6} \mathrm{Li} /{ }^{7} \mathrm{Li}$ isotopic ratio. In this way, analysis of the Li I line profiles in magnetic $\mathrm{CP}$ stars can give new challenges to the theoretical calculations of diffusion in the presence of magnetic fields and to the understanding of the dynamic processes occuring in the stellar regions where 
the lithium lines are formed. The main difficulty in the development of theoretical models explaining such peculiar behaviour of the lithium lines in the spectra of CP stars has been the scarcity of the observations in the Li I $6708 \AA$ spectral region. In order to clarify the situation, the international Project "Lithium in the cool CP stars with magnetic fields" was started in 1996.

The main goals of the Project are:

- to collect new observations in the spectral region of the Li I $6708 \AA$ line;

- to study the Li I $6708 \AA$ line behaviour with rotating phase;

- to study the connection of the lithium distribution on the surfaces of CP stars and the structure of magnetic fields;

- to create a database of the CP stars spectra in the region of the Li I $6708 \AA$ and 6104 A lines;

- to search for physical mechanisms which could explain the anomalous behaviour of this line on the surface of magnetic CP stars.

\section{Main stages of the Project realization}

The first steps of the Project realization consisted in the systematical observations of two CP stars $\beta$ CrB and $\gamma$ Equ in the spectral region of the Li I $6708 \AA$ line carried out by N. Polosukhina with the ZTSh $2.6 \mathrm{~m}$ telescope of the Crimean Astrophysical Observatory (CrAO). These observations showed a strong and unvariable Li I $6708 \AA$ line in the spectrum of $\gamma$ Equ and the variability of this line with the rotation phase in the spectrum of $\beta \mathrm{CrB}$ (Polosukhina \& Lyubimkov 1995). This study, together with the results obtained by Hack et al. (1997), was the base of the Project adopted during the Meeting on Ap stars held in Slovakia in 1996.

The next series of observations were carried out by P. North in 1998 with the $1.4 \mathrm{~m}$ ESO CAT telescope with high spectral resolution $(R=100000)$. The spectra of $12 \mathrm{CP}$ stars in the Li I $6708 \AA$ spectral region were obtained. The main result of these observations was the discovery of the variability, both in intensity and in wavelength, of the Li I $6708 \AA$ line profile with the rotational phase in the spectra of HD 83368 and HD 60435, two roAp stars. These variations were interpreted as the existence of two $\mathrm{Li}$ spots on the surfaces of these stars (North et al. 1998). Fig. 1 shows the variations of the equivalent widths and radial velocities of the Li I $6708 \AA$ lines in the spectra of HD 83368 and HD 60435 . Model of the oblique rotator explaining the behaviour of the Li I line is shown in Fig. 2 (Polosukhina et al. 1999).

\section{Determination of the Li-spots parameters}

The next important result in the study of HD 83368 and HD 60435 was the determination of the parameters of the "lithium spots" and their Li abundances using the codes STARSP and ROTATE written by V. Tsymbal. The best agreement between calculated and observed profiles of the Li I $6708 \AA$ line for all phases was achieved for the following parameters:

HD 83368: $\left(T_{\text {eff }}=7750 \mathrm{~K}, \log g=4.0, i=90^{\circ}, v_{\mathrm{e}}=35 \mathrm{~km} \mathrm{~s}^{-1}\right.$, and photospheric Li abundance $\left.\log \varepsilon_{\mathrm{ph}}(\mathrm{Li})=1.8 \dagger\right)$

Spot 1: $l_{1}=173^{\circ} \pm 6^{\circ}, \varphi_{1}=0^{\circ} \pm 6^{\circ}, R_{1}=33^{\circ} \pm 6^{\circ}, \log \varepsilon_{1}(\mathrm{Li})=3.6 \pm 0.2$

Spot $2: l_{2}=337^{\circ} \pm 6^{\circ}, \varphi_{2}=0^{\circ} \pm 6^{\circ}, R_{2}=35^{\circ} \pm 6^{\circ}, \log \varepsilon_{2}(\mathrm{Li})=3.5 \pm 0.2$

$\dagger$ Where $\log \varepsilon(\mathrm{X})=\log \left(N_{\mathrm{X}} / N_{\mathrm{H}}\right)+12.0$ 


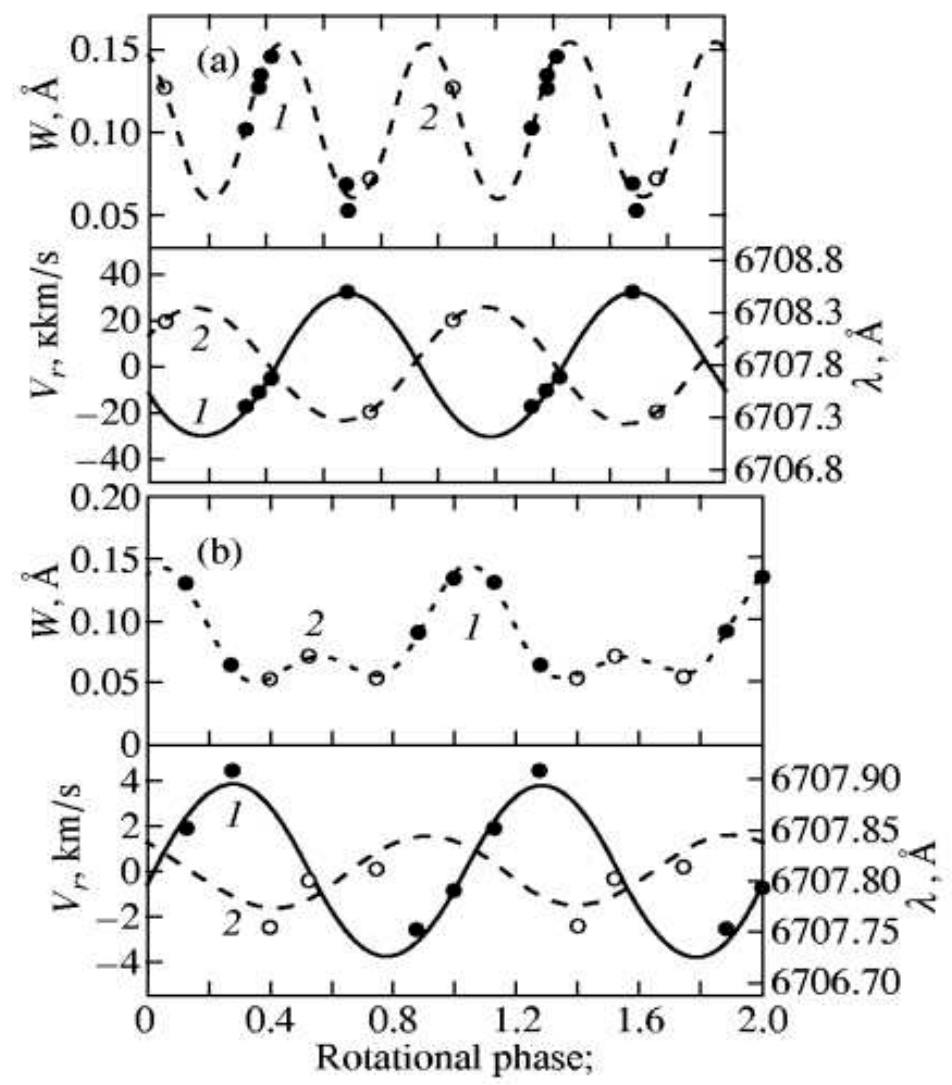

Figure 1. Variations of the equivalent width and radial velocity of the Li I $6708 \AA$ line in HD 83368 (a) and HD 60435 (b). Dark circles - spot 1, open circles - spot 2. The curves show the fit by a sinusoidal law.

HD 60435: $\left(T_{\text {eff }}=8250 \mathrm{~K}, \log g=4.5, i=47^{\circ}\left(133^{\circ}\right), v_{\mathrm{e}}=15 \mathrm{~km} \mathrm{~s}^{-1}\right.$, and photospheric $\mathrm{Li}$ abundance $\left.\log \varepsilon_{\mathrm{ph}}(\mathrm{Li})=1.8\right)$

Spot 1: $l_{1}=11^{\circ} \pm 6^{\circ}, \varphi_{1}=-15^{\circ} \pm 5^{\circ}, R_{1}=44^{\circ} \pm 3^{\circ}, \log \varepsilon_{1}(\mathrm{Li})=3.8 \pm 0.2$

Spot 2: $l_{2}=205^{\circ} \pm 10^{\circ}, \varphi_{2}=15^{\circ} \pm 5^{\circ}, R_{2}=40^{\circ} \pm 7^{\circ}, \log \varepsilon_{2}(\mathrm{Li})=2.7 \pm 0.2$

Comparison of observed and calculated with these spot parameters profiles of the $\mathrm{Li}$ I $6708 \AA$ line is shown on Figs. 3 and 4 .

\section{Monitoring of HD 83368 with the $1.52 \mathrm{~m}$ telescope and FEROS in ESO}

The above results gave an impulse for new monitoring of the star HD 83368 in the broad spectral region $(3650-9270 \AA)$ covering Li I resonance and secondary lines at $6708 \AA$ and $6104 \AA$ with the FEROS spectrograph at the $1.52 \mathrm{~m}$ telescope in ESO, La Silla, in December 2000 (see Fig. 5). Surface distribution of the Li, as well as of other elements, was obtained using the Doppler Imaging code INVERS12 (Kochukhov et al. 2004). The Doppler Imaging analysis confirmed that Li is concentrated in two small spots near the poles of the magnetic field. 

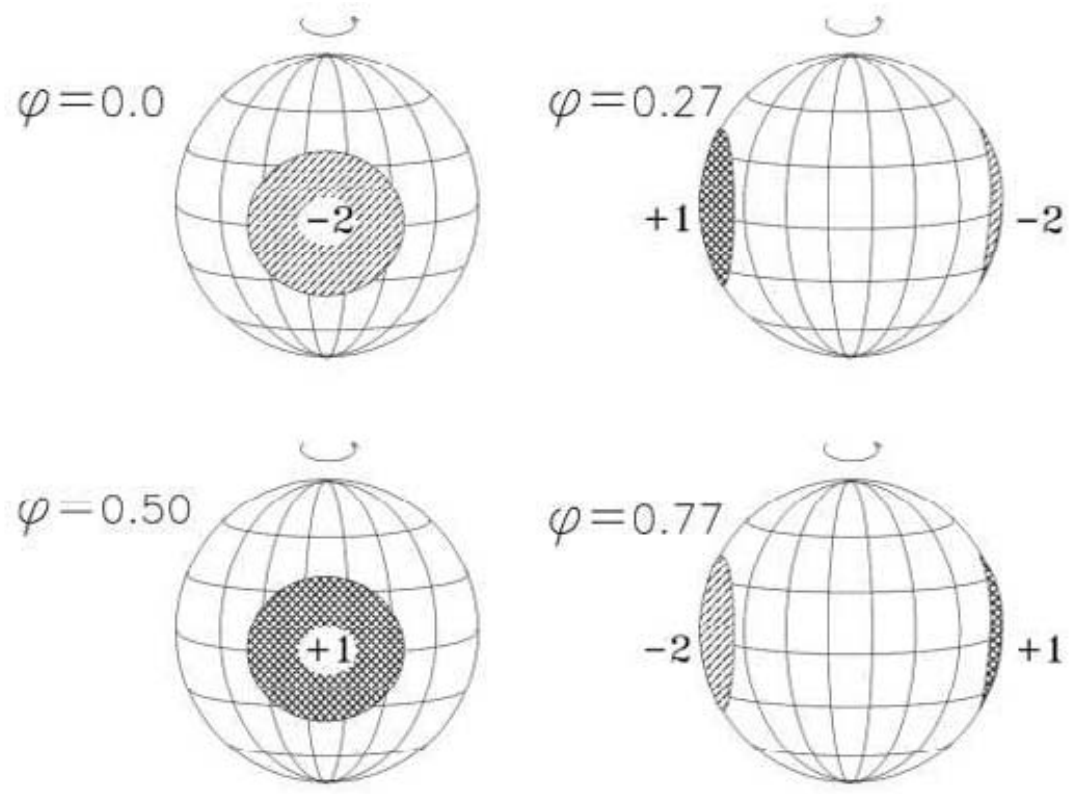

Figure 2. Spherical model of the surface of HD 83368 with two lithium spots (1 and 2).

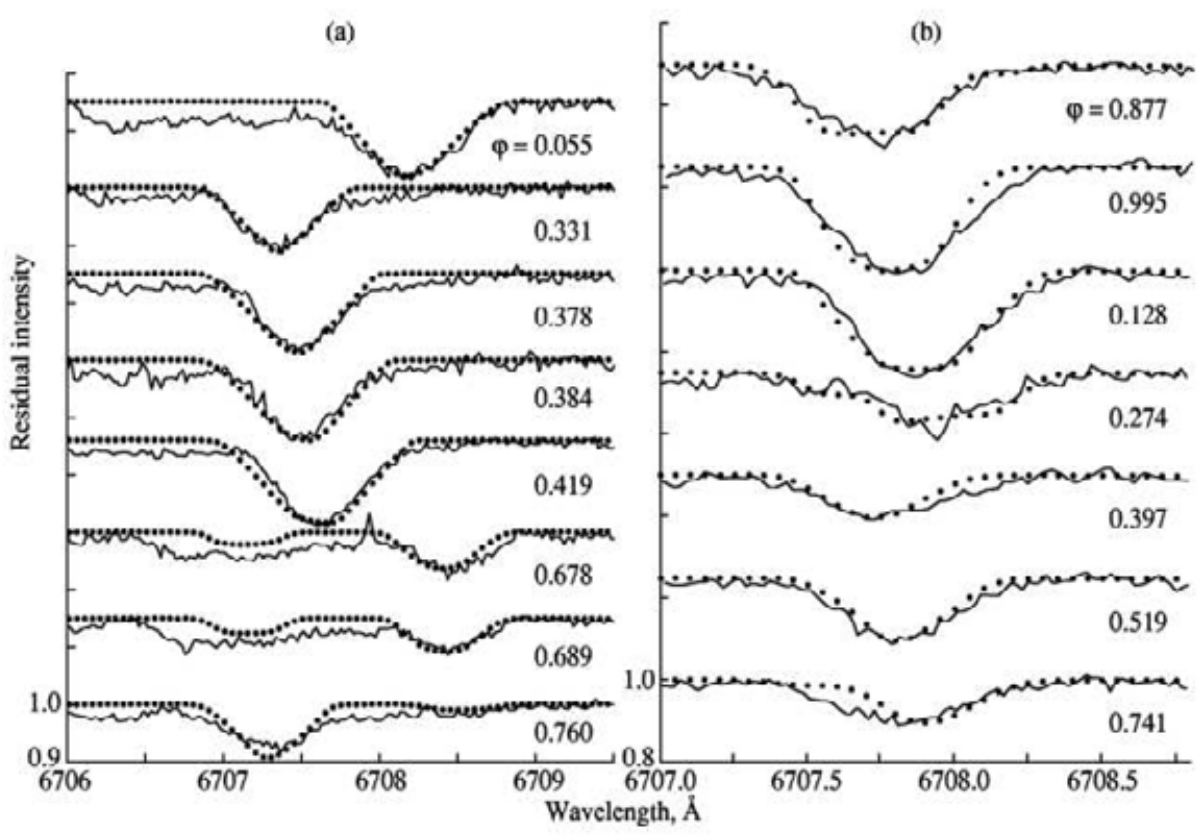

Figure 3. Observed (solid lines) and calculated (dots) profiles of the Li I $6708 \AA$ line for different rotational phases. (a) HD 83368 (b) HD 60435

\section{CP stars observations at Mount Stromlo Observatory}

High-resolution spectra $(R=88000)$ of $12 \mathrm{CP}$ stars were obtained during the observing run in September-October 2001 with the 74-inch telescope and echelle spectrograph of the Mount Stromlo Observatory (Polosukhina et al. 2003).

We detected a strong Li I line in the spectrum of HD 208217 obtained on September 


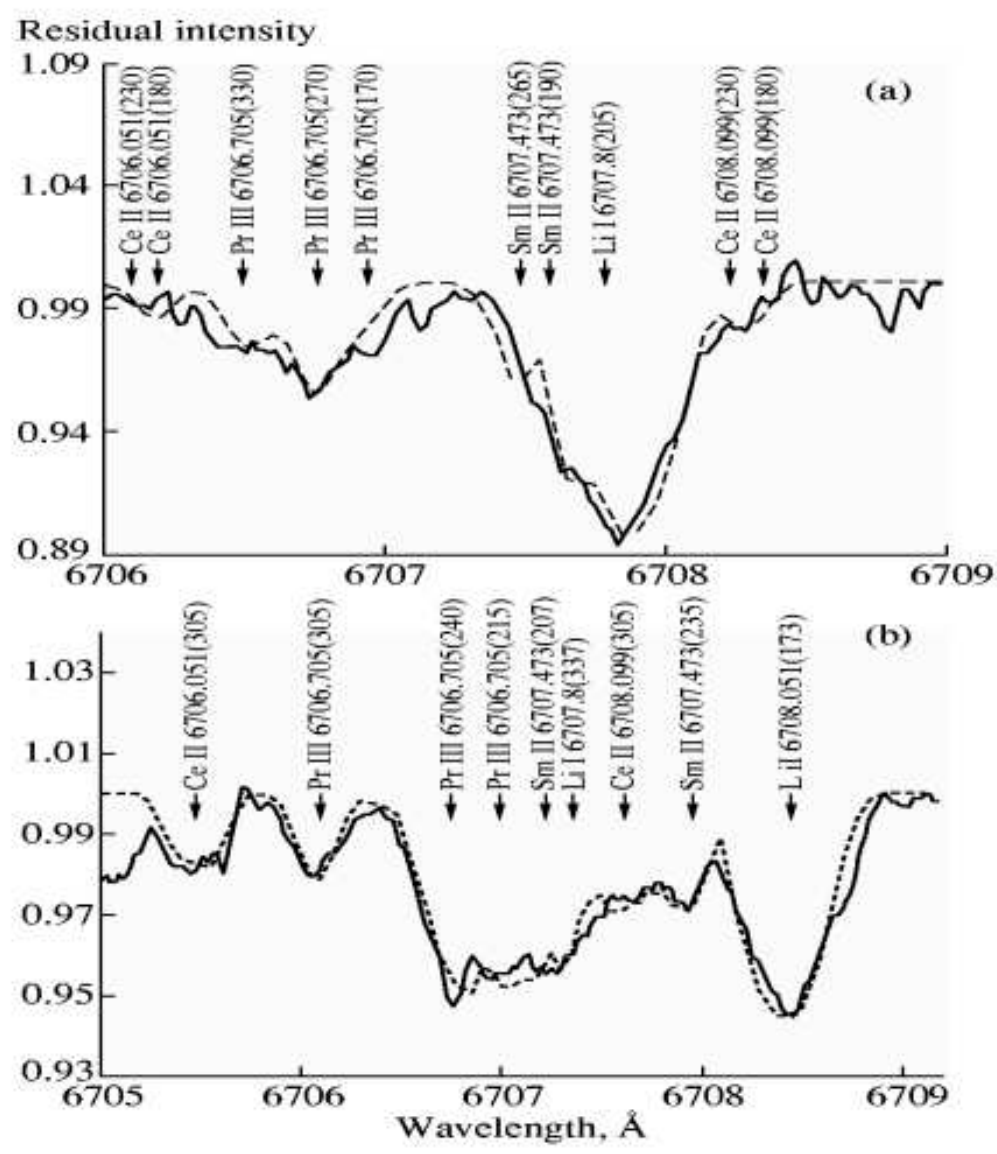

Figure 4. Effects of spot structure on line profiles. Observed (solid lines) and calculated (dashed lines) Li I $6708 \AA$ line profiles are shown. (a) HD 60435 ( $\varphi=0.743)$ (b) HD 83368 ( $\varphi=0.689$ )

27, 2001. In the paper of Faraggiana et al. (1996) this star was classified as a star with a very weak absorption at $6708 \AA$. This fact points to a strong variability of the Li I line with the rotation phase. Monitoring of HD 208217 is needed.

The most interesting result was obtained for the star HD 3980. This star shows strong variations of position and intensity of the Li I $6708 \AA$ line with the rotation phase. Detailed analysis of this star is presented in Drake et al. (2004) in this Symposium.

\section{CP stars observations with the $6 \mathrm{~m}$ BTA telescope at SAO}

Recently, in April 8-9, 2004, a new set of observations has been carried out with the $6 \mathrm{~m}$ BTA telescope of the Special Astrophysical Observatory of the Russian Academy of Sciences (SAO). Preliminary results of these observations are presented in Polosukhina et al. in this Symposium.

\section{Analysis of the Przybylski's star spectrum in the Li I $6708 \AA$ spectral region}

A detailed analysis of the spectra of the unique roAp star HD 101065 (Przybylski's star) near the resonance Li I $6708 \AA$ doublet has been carried out by Shavrina et al. 


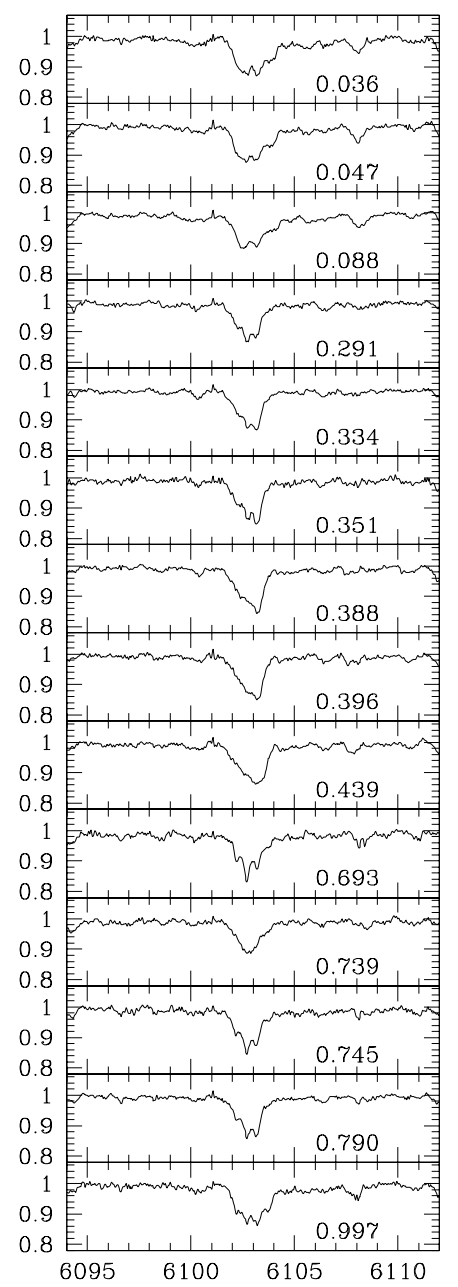

(a)

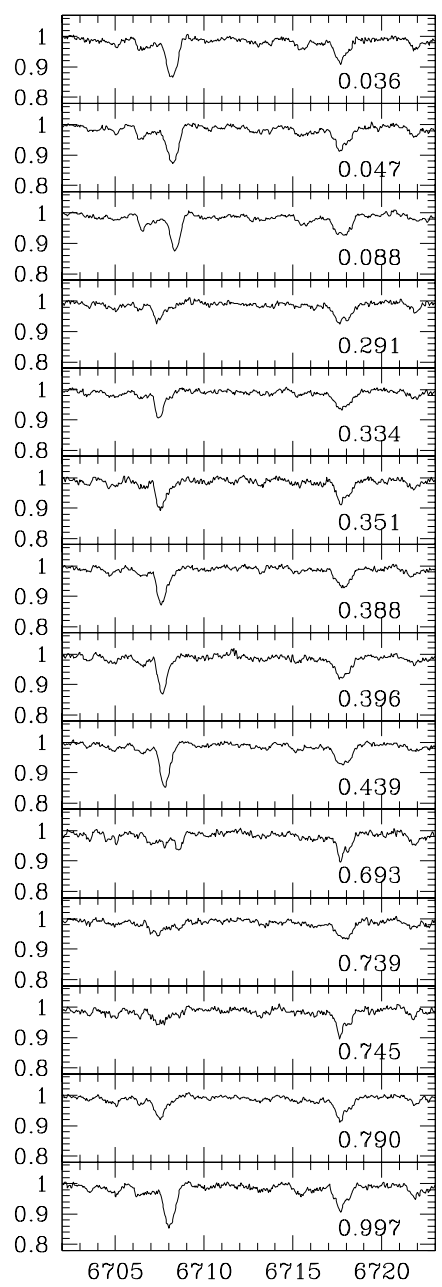

(b)

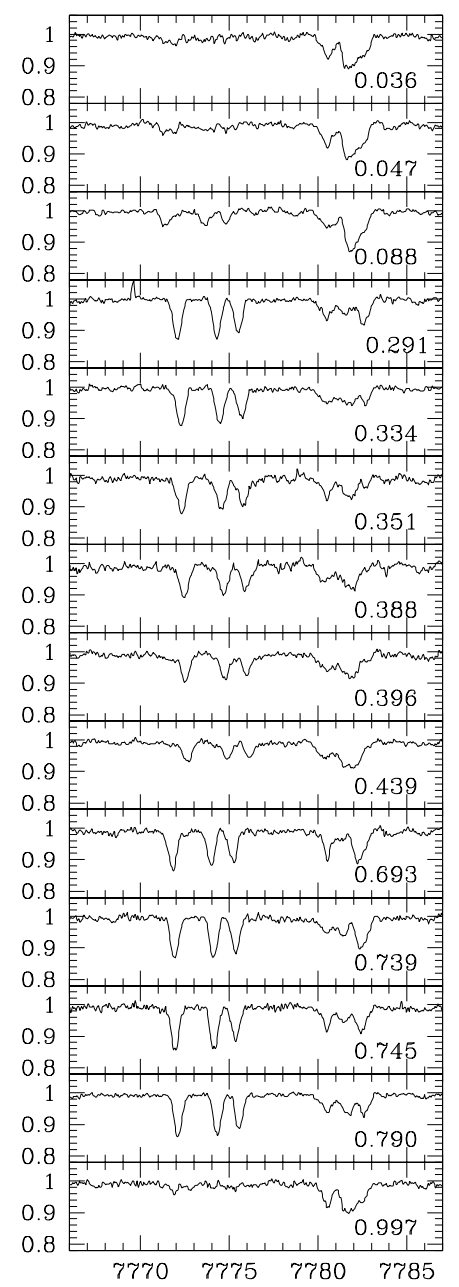

(c)

Figure 5. Spectra of the roAp star HD 83368 in the spectral regions of the Li I lines at $6104 \AA$ and $6708 \AA$ (a and b) and $\mathrm{O}$ i triplet at $\sim 7770 \AA$ (c) obtained with the $1.52 \mathrm{~m}$ telescope with FEROS, ESO. The rotational phases were calculated using the period of 2.851976 days (Kurtz et al. 1997).

(2003). In this work, authors used the most complete line list including all possible transitions between REE levels of the NIST database. Synthetic spectra calculations proved that $\mathrm{Li}$ lines are present in the range $\lambda 6707.72-6708.02 \AA$ and that the resulting $\mathrm{Li}$ abundance is $3.1 \mathrm{dex}$. High isotopic ratio of ${ }^{6} \mathrm{Li} /{ }^{7} \mathrm{Li}=0.33$ might be considered as indication of spallation reactions on the surface of this star. The fitting of observed spectrum is presented in Fig. 6 .

\section{Conclusions}

We have presented some results of the Li I $6708 \AA$ line analysis in the spectra of CP stars based on the series of observations carried out with the telescopes $2.6 \mathrm{~m}$ ZTSh (CrAO), 1.4m CAT (ESO), 1.52m with FEROS (ESO), and 74-inch (Mount Stromlo Observatory) in the framework of the Project "Lithium in the cool CP stars with magnetic fields": 


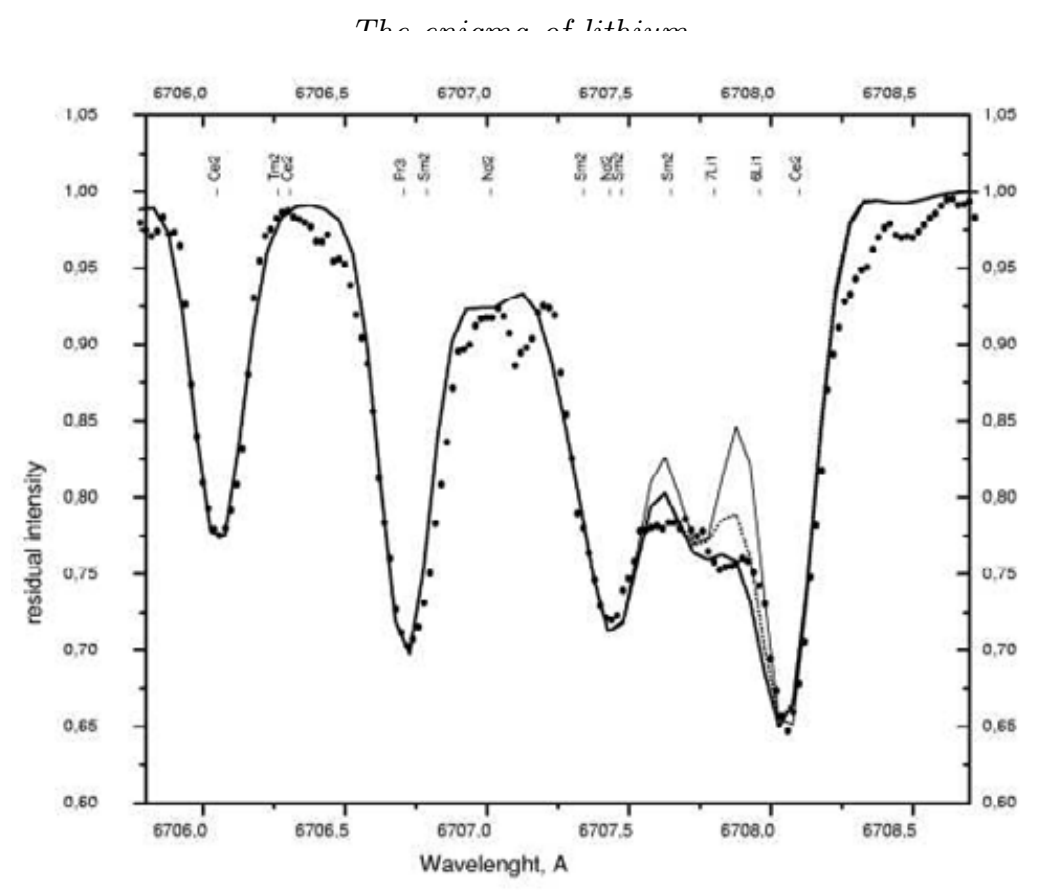

Figure 6. The fitting of observed spectrum of HD 101065 near $6708 \AA$. Dots - observed spectrum, dashed line - calculated spectrum with ${ }^{7} \mathrm{Li}$ lines only, thick line spectrum with the ratio ${ }^{6} \mathrm{Li} /{ }^{7} \mathrm{Li}$ $=0.33$. The thin line corresponds to a spectrum calculated without $\mathrm{Li}$ but with $\mathrm{Sm}$ II line $6707.799 \AA$. The positions of the lines which are the main contributors in absorption are marked at the top of the figure.

- The first indications of variations of the Li I $6708 \AA$ line with rotation phase were obtained in CrAO (2.6 m ZTSh telescope) for $\beta$ CrB.

- Strong evidence of an inhomogeneous distribution of Li (lithium spots) on the surfaces of roAp stars HD 83368 and HD 60435 was obtained using the $1.4 \mathrm{~m}$ CAT telescope (ESO).

- A good correlation between the positions of the Li spots, magnetic field and brightness indicates a connection between the magnetic field configuration, the local structure of the atmosphere and the distribution of the chemical anomalies.

- Preliminary analysis of the Li spots and determination of their parameters, such as size, position and Li abundance, was performed with the ROTATE code written by V. Tsymbal.

- Monitoring of HD 83368 with the $1.52 \mathrm{~m}$ telescope and spectrograph FEROS (ESO) in the Li I $6708 \AA$ line permitted to carry out Doppler Imaging analysis of the Li surface distribution (Kochukhov et al. 2004). Good agreement with the results of direct modelling was obtained.

- Preliminary analysis of the slowly rotating CP stars HD 137949, HD 134214, and HD 166473 was performed by J. Zverko and J. Žižňovský. They determined the Li abundance in these stars and showed that these stars have anomalously high isotopic ratio ${ }^{6} \mathrm{Li} /{ }^{7} \mathrm{Li}$ (Zverko et al. 2000).

- New testing observations of twelve CP stars in Mount Stromlo Observatory with the 74-inch telescope (2001) permitted us to find new CP stars exhibiting the Li I $6708 \AA$ line. Monitoring of some CP stars resulted in the discovery of a new Li-spotted rotator - the star HD 3980 (Drake et al. 2005). 


\section{References}

Babel, J., Michaud, G. 1991, A\&A, 248, 155

Babel, J. 1993, in ASP Conf. Ser. 44, Peculiar versus Normal Phenomena in A-type and Related Stars, eds. M.M. Dworetsky, F. Castelli, \& R. Faraggiana, 458

Drake, N.A., Polosukhina, N.S., de la Reza, R., Hack, M., 2005, These Proceedings, FP11

Faraggiana, R., Gerbaldi,M., Delmas, F. 1966, Ap\&SS 238, 169

Hack, M., Polosukhina, N., Malanushenko, V. \& Castelli, F. 1997, A\&A A 319, 637

Kochukhov, O., Drake, N.A., Piskunov, N., de la Reza, R. 2004, A\&A (in press)

Kurtz, D.W., van Wyk, F., Roberts, G., Marang, F., et al. 1997, MNRAS, 287, 69

North, P. Polosukhina, N., Malanushenko, V., Hack, M. 1998, A\&̊A, 333, 644

Polosukhina, N.S., Lyubimkov, L.S. 1995, Mm. SAI, 66, 361

Polosukhina, N.S., Kurtz, D., Hack, M., North, P., Ilyin, I., Zverko, J., Shakhovskoy, D. 1999, $A \mathscr{G} A, 351,283$

Polosukhina, N.S., Shavrina, A.V., Hack, M., Khalack, V., Tsymbal, V., North, P. 2000, A\&A, 357,920

Polosukhina, N.S., Drake, N.A., Hack, M., de la Reza, R., Wood, P.R., Shavrina, A.V. 2003, ASP Conf. Ser., IAU Symp. 210 Modelling of Stellar Atmospheres, eds. N.E. Piskunov, W.W. Weiss, and D.F. Gray (astro-ph/0301150)

Shavrina, A.V., Polosukhina, N.S., Zverko, J., Khalak, V., Tsymbal, V.V., Žižňovský, J. 2001, Astron. Rep. 10, 784

Shavrina, A.V., Polosukhina, N.S., Pavlenko, Ya.V., Yushchenko, A.V., Quinet, P., Hack, M., North, P., Gopka, V.F., Zverko, J., Žižňovský, J., Veles, A. 2003, A\&A, 409, 707 